(C) 1984. The Genetical Society of Great Britain

\title{
GENE TRANSFER IN NICOTIANA RUSTICA BY MEANS OF IRRADIATED POLLEN II CYTOGENETICAL CONSEQUENCES
}

\author{
C. P. WERNER, I. M. DUNKIN, M. A. CORNISH AND G. H. JONES \\ Department of Genetics, University of Birmingham, Birmingham B15 2TT, England
}

Received 8.vi.83

\begin{abstract}
SUMMARY
Pollen from Nicotiana paniculata and the $V_{12}$ variety of $N$. rustica was irradiated with a range of high doses of $\gamma$-rays up to $100 \mathrm{Krads}$. Both kinds of pollen were used to pollinate the $V_{27}$ variety of $N$. rustica. Radiation treatments above $30 \mathrm{Krads}$ gave no viable seed. A cytological examination of the $M_{1}$ progeny from the $20 \mathrm{Krad}$ treatments of both crosses revealed conventional radiation damage in the form of losses of whole chromosomes and parts of chromosomes, and rearrangements. The plants possessed hybrid or aberrantly hybrid phenotypes. It was concluded that they were the products of a conventional fertilisation mechanism rather than the gene transfer mechanism proposed by Pandey (1980). The expression of mutational damage can probably account for most of the maternal trends observed in the intervarietal $\mathrm{M}_{2}$ of $N$. rustica examined previously, although post-meiotic selection may also play a role.
\end{abstract}

\section{INTRODUCTION}

Pandey $(1975,1978,1980)$ observed that the $M_{1}$ progeny resulting from crosses in the genus Nicotiana following irradiation of the pollen with $\gamma$-rays differed genetically from the unirradiated hybrids. Although some of the irradiated progeny had inherited paternal flower colour or self-incompatibility alleles, they are reported to have appeared otherwise maternal. To explain this Pandey proposed a mechanism of gene transfer, whereby fragments of pulverised paternal chromosomes are incorporated into the maternal genome of the ovule. Diploidy and fertility are then restored either by parthenogenetic doubling of the maternal genome, or by a second fertilisation by the unirradiated, incompatible pollen.

A similar trend towards the maternal phenotype was found in the $\mathbf{M}_{2}$ progenies derived from the irradiated, intervarietal crosses in Nicotiana rustica described in the previous paper of this series (Caligari, Ingram and Jinks, 1981). Results based on the measurement of a number of characters led Caligari et al., to conclude that Pandey's gene transfer mechanism could also be operating in this species. They also suggested, however, that the observations could result from the expression of induced mutations or large-scale inactivations of the paternal genome following an otherwise normal fertilisation. A similar explanation has been proposed by Engvild (1981) to explain some of Pandey's results.

More recently, work on barley, Hordeum vulgare, (Powell, Caligari and Hayter, 1983) and on wheat Triticum aestivum, (Snape, Parker, Simpson, Ainsworth, Payne and Law, 1983) has provided further evidence of the 
effects of pollen irradiation on the derived generations. In barley Powell et al., found a similar dose-related effect to that found in $N$. rustica by Caligari et al. At the highest dose of irradiation four $\mathrm{M}_{1}$ barley plants gave rise to $\mathrm{M}_{2}$ families showing close resemblances to the maternal parent for the three characters measured. On the other hand, the phenotypes of the two $\mathrm{M}_{2}$ families of wheat examined by Snape et al., showed trends towards the maternal parent for one quantitative character but towards the paternal parent for a second character. Snape et al. concluded that they were observing a mixture of the effects of radiation damage and of a greater than expected representation of maternal alleles in the $\mathbf{M}_{2}$. Cytological evidence, however, led them to reject Pandey's proposed mechanism of gene transfer to explain this maternal bias. Instead they proposed that their $M_{1}$ progeny were the products of conventional fertilisations by pollen carrying some radiation damaged chromosomes. This radiation damage then caused a disturbance at $M_{1}$ meiosis which resulted either in the direct loss of paternal chromosomes or in a loss of function of the gametophytes carrying them. A similar gametophytic selection mechanism has been proposed by Donini et al. (1970) and Devreux et al. (1972) to account for disturbed segregations in the $\mathbf{M}_{2}$ generations of barley following pollen irradiation.

In order to distinguish between the various mechanisms which have been proposed we have undertaken a cytological examination of both interand intraspecific $M_{1}$ progeny in the Nictotiana genus. We chose an interspecific cross between species of different chromosome number $(N$. rustica, $2 n=48$, and one of its diploid progenitors, $N$. paniculata, $2 n=24$ ) since in this case the consequences of Pandey's mechanism differ markedly from those of a more normal fertilisation. We also examined progeny from the same irradiated, intervarietal cross of $N$. rustica used by Caligari et al., (loc cit.) in an attempt to explain their observations. The results of these investigations are reported here.

\section{MATERIALS AND METHODS}

An inbred variety of Nicotiana rustica, $V_{27}$, was used as the female parent in both the interspecific crosses and the intraspecific crosses. Pollen adhering to freshly dehisced anthers of $N$. paniculata and of $N$. rustica $V_{12}$ was irradiated with $\gamma$-rays at doses of 10,20,30,50 and $100 \mathrm{Krads}$ and used to pollinate emasculated $V_{27}$ flowers. The unirradiated crosses were also made.

The resultant seedlings were grown in the glasshouse in double peat pots. Actively growing root tips were collected for mitotic analysis from the moist cavity between the two pots. Root tips were pretreated for 3 hours at $19^{\circ} \mathrm{C}$ in a saturated solution of 8 -hydroxyquinoline (Gerstel, Burns and Burk, 1978) and then stored in Carnoy's fixative, in a freezer, overnight. The fixed material was hydrolysed for $5-10$ minutes in $5 \mathrm{~N} \mathrm{HCl}$ at room temperature. After washing, root tips were stained for a few minutes in Feulgen's reagent and squashed in lactopropionic orcein.

The cytological assessment of each plant was obtained from a number of well spread cells, often taken from more than one root tip. In each case this permitted a reliable count of the number of chromosomes and, in some cases, the identification of gross chromosomal abnormalities. 


\section{Results}

\section{(i) The interspecific cross}

The numbers of viable, hybrid seed produced per capsule decreased as the radiation dose increased. No viable seed were obtained from any of the pollinations following the 50 and $100 \mathrm{Krad}$ treatments, whilst the $30 \mathrm{Krad}$ treatment gave only 2 per cent of the seed yield of the unirradiated pollen. Efforts were concentrated on the $20 \mathrm{Krad}$ treatment, which was the highest dose to give a reasonable number of seed and the highest dose used by Caligari et al.

Examination of root tip mitoses in 9 unirradiated hybrid plants revealed the expected, triploid, chromosome number of 36, including 5 satellited chromosomes. $N$. rustica and $N$. paniculata have 3 and 2 pairs of satellited chromosomes respectively. There are no other detectable morphological differences between the chromosomes of the two parents. It should also be noted here that their normal karyotypes do not include any telocentric chromosomes.

The cytological composition of $56 \mathrm{M}_{1}$ progeny from the $20 \mathrm{Krad}$ treatment are given in table 1 . Half the plants possessed karyotypes indistinguishable at mitosis from the normal, hybrid karyotype. The other half showed evidence of chromosomal damage, chromosomal loss, or both. Thus, 17 plants had lost a single chromosome, the remaining 35 chromosomes appearing undamaged. Other plants possessed chromosomes which consistently appeared shorter than any in the normal karyotype, indicating the loss of part of a chromosome or of a whole arm (plate 1a). The most cytologically aberrant plant in the family had only 33 chromosomes, one of which was telocentric.

TABLE 1

The cytological composition of the 56 interspecific $20 \mathrm{Krad} M_{J}$ (N. rustica $\times$ N. paniculata) plants (Key to abnormal chromosomes: T-telocentric, $R$-reduced, I-increased)

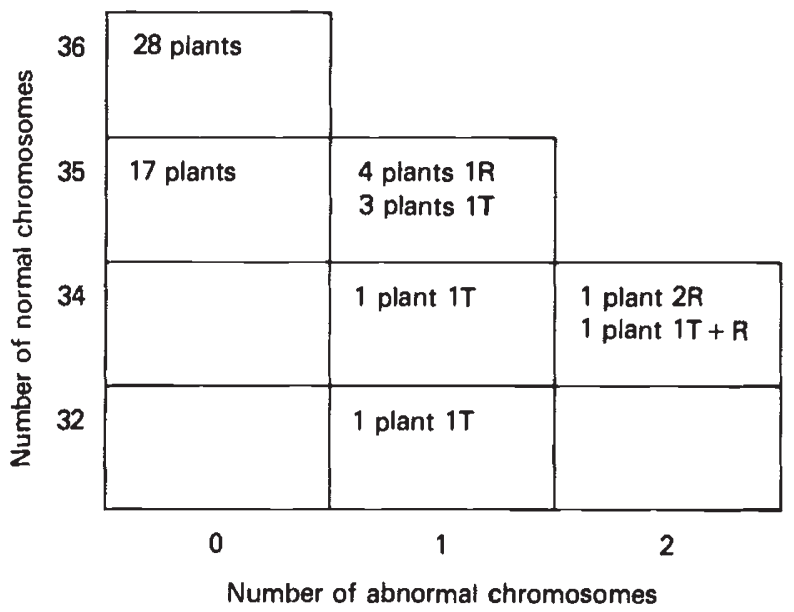

A morphological assessment of the 56 plants revealed a similar pattern. The normal hybrids were roughly intermediate in form to the two parents. The $M_{1}$ plants, whilst showing more variation, were in general similar in 


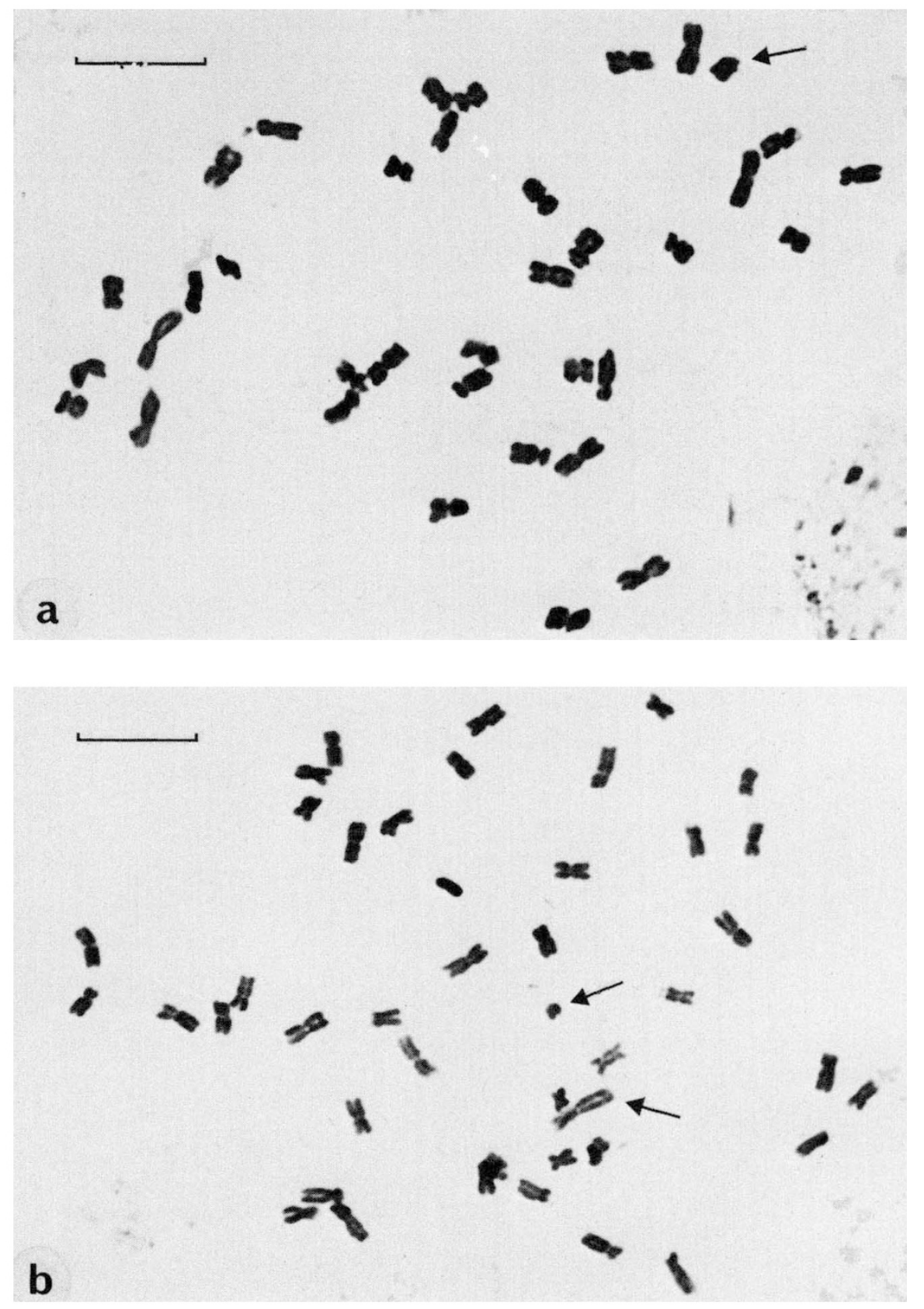

Plate 1. Chromosome complements of $M_{1}$ plants at mitosis. (a) Interspecific $M_{1}$ (20 Krad) with 36 chromosomes, one of which is telocentric; (b) Intraspecific $M_{1}$ (10 Krads) with 44 chromosomes including one telocentric and one increased in length. The bars represent $10 \mu$. 
phenotype to the normal hybrid. No maternal trends were apparent, although a number of plants showed morphological aberrations ranging from minor leaf abnormalities or slight reductions in growth rate to more severe morphological and developmental defects. As expected, the morphologically more aberrant plants tended to be those with missing or damaged chromosomes. 11 of the 56 plants also lacked the dominant green flower and plant colour present in the normal hybrid and originating from the pollen parent, $N$. paniculata.

An examination of the meiotic configurations of $920 \mathrm{Krad} M_{1}$ plants gave additional evidence of chromosomal damage. Whilst 6 plants displayed the normal 12 bivalents and 12 univalents found in the unirradiated hybrid, the other 3 consistently produced quadrivalents, revealing reciprocal translocations not apparent at mitosis. Some plants showed an increased frequence of rod bivalents, indicating a reduction in chiasma frequency as a result of radiation damage.

In addition to the $20 \mathrm{Krad} \mathrm{M}_{1}$ progeny plants from both the $10 \mathrm{Krad}$ and $30 \mathrm{Krad}$ treatments were examined. These showed a similar range of deviations from the normal hybrid, both cytologically and morphologically. A dose effect was apparent, with an increase in the frequency of both kinds of abnormality as the radiation dose increased. One plant from the $30 \mathrm{Krad}$ treatment had 37 chromosomes, one more than the normal hybrid. A further two plants from this treatment had 48 chromosomes and appeared phenotypically identical to the maternal parent. These plants could have been either matromorphs or self contaminants.

\section{(ii) The intraspecific cro is}

The seed yield in the cross between the two varieties of $N$. rustica $\mathrm{V}_{27} \times \mathrm{V}_{12}$, varied similarly with dose to that of the interspecific cross and

TABLE 2

The cytological compositon of the 35 intraspecific $20 \mathrm{Krad} M_{1}\left(V_{27} \times V_{12}\right)$ plants

(Key to abnormal chromosomes: $T$-telocentric, $R$-reduced, I-increased)

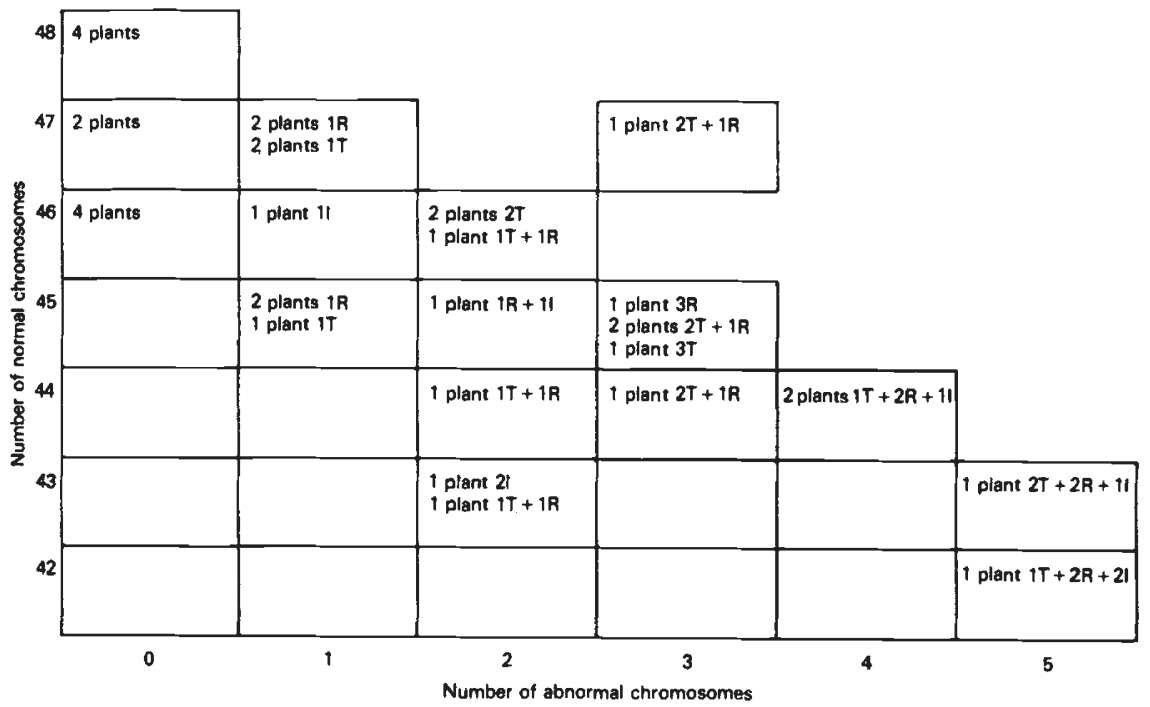


more attention was again given to the $20 \mathrm{Krad}$ treatment, for the same reasons. Mitotic karyotypes were examined in $3520 \mathrm{Krad} \mathrm{M}_{1}$ plants (table 2). In this case the majority of plants showed evidence of chromosomal loss or damage. Only 5 plants had apparently undamaged karyotypes of 48 chromosomes. Most plants had some chromosomes reduced in length as a result of the radiation treatment, whilst 7 plants also had one or two chromosomes consistently longer than any found in the normal karyotype. One plant had 50 chromosomes, 3 of which were reduced in length, but it was not phenotypically unusual.

Although some plants in this sample were similar in phenotype to the normal $F_{1}$ hybrid, many showed the expected deleterious effects of aneuploidy and structural chromosome damage, and a few were extremely aberrant. Fertility was reduced in almost all cases and some plants were completely sterile. Multivalent formation was apparent in the meiotic configurations of some of the plants examined.

Following pollen irradiation at $30 \mathrm{Krads} 7$ maternal haploids were found amongst 110 plants produced from 39 pollinations. There were also 8 plants which, like the two plants from the interspecific cross, were fully fertile and identical to the maternal parent in both phenotype and karyotype. The remainder showed the same range of radiation induced variation found in the $20 \mathrm{Krad}$ treatment.

\section{Discussion}

The mechanism of gene transfer as proposed by Pandey cannot explain the results presented here. If a process of fragment incorporation and parthenogenic chromosome doubling were occurring following these two irradiated crosses, we should expect all the progeny produced from both crosses to possess the normal, maternal karyotype. We should also expect them to be morphologically more like $V_{27}$ than the normal hybrid and to be fully fertile. This is clearly not the case. Instead, both sets of progeny appear to have resulted from conventional fertilisations, albeit with radiation induced damage. The triploid karyotypes of many of the interspecific progeny show that after $20 \mathrm{Krads}$ of $\gamma$-radiation the chromosomes of some pollen grains of $N$. paniculata remain essentially intact and unfragmented. Other grains, although possessing damage, which results in deletions, interchanges and the loss of whole chromosomes, are still capable of successful fertilisations to form viable zygotes. This conclusion is also supported by the hybrid or aberrantly hybrid phenotypes of these individuals.

The cross between the two varieties of $N$. rustica, despite being between parents with identical karyotypes, leads us to the same conclusion. In this cross nearly all the progeny show cytological evidence of the effects of radiation. This indicates that they too are the products of conventional fertilisations by irradiated pollen grains rather than of the incorporation of paternal fragments into a doubled maternal complement. Although many of these plants differ morphologically from the unirradiated hybrid they are not more maternal in appearance, though some individual characters may be. They are merely aberrant, displaying the typical deleterious effects of mutations, deletions and aneuploidy. They also show various degrees of sterility as a result of the radiation damage. Similar results were obtained from previous experiments using irradiated pollen in $N$. rustica (Ivanov, 1938) and in N. tabacum (Goodspeed, 1929, Pandey and Phung, 1982). 
The cytological abnormalities observed here are not qualitatively different from those found in numerous experimental studies of radiation effects, despite the very high radiation doses employed. It seems likely that our viable $M_{1}$ zygotes resulted from fertilisations by the least damaged pollen grains. The majority of pollen grains were probably more severely damaged and, therefore, failed either to effect a fertilisation or to produce a viable zygote. The additional chromosomes found in two plants (one from each cross) were probably the results of radiation induced disturbances of the mitotic division that occurs in the pollen tube prior to fertilisation. Cytological abnormalities were more frequent in the intervarietal progeny than in the interspecific progeny. This may indicate that the diploid (polyhaploid) pollen of $N$. rustica is more tolerant of aberrations than the haploid pollen of $N$. paniculata, or that the tetraploid zygotes are more tolerant than the triploid zygotes.

Since the intervarietal cross of $N$. rustica examined here was the same as that used by Caligari et al., we are now able to offer a re-interpretation of their findings. Their original $\mathrm{M}_{1}$ plants must have been the more fertile individuals from families of hybrid plants similar to ours. They would, therefore, have differed from the $F_{1}$ by possessing paternally derived chromosomes suffering radiation damage ranging from large deletions and interchanges, visible at mitosis and meiosis, to smaller changes undetectable cytologically and possibly including point mutations. At least some of this damage is likely to have been inherited by the $\mathrm{M}_{2}$ generation in which Caligari et al. observed maternal trends. Now, induced mutations, whether point mutations or deletions, tend to result in the reduction or loss of function of the genes affected, which in turn tends to have a deleterious effect on the phenotype. We might, therefore, expect $\mathrm{M}_{2}$ plants which had inherited induced mutations to show a general loss of vigour and, in particular, to be smaller on average than the $F_{2}$ generation. This is exactly the situation that Caligari et al., observed. Their $\mathrm{M}_{2}$ plants were, on average, shorter than the $F_{2}$ for all three of the height characters presented and, since the maternal parent $V_{27}$ is a short variety, the $M_{2}$ showed a closer resemblance to the maternal parent than did the $F_{2}$. However, if the reduction in height is entirely a result of the expression of mutational damage then this maternal resemblance is coincidental. If the reciprocal cross were to be made then we should still expect mutational damage to lead to a reduction in height in the $\mathrm{M}_{2}$, but in this case the resemblance would be to the paternal parent. This was the case found by Snape et al., when a short variety of wheat was used as the irradiated pollen parent in a cross with a taller variety.

Although we believe that this expression of mutational damage in the $\mathrm{M}_{2}$ generation could account for most of the trends observed by Caligari et al., we cannot, as yet, rule out the possibility that postmeiotic selection similar to that proposed by Snape et al. may also play an important role. It seems unlikely to us that the meiotic process itself could act as a selective agent (Snape et al., 1983), but it is possible that some of the mutations and deletions carried by the paternal chromosomes may be lethal in the gametes or the resultant zygotes. Selection could act at these stages against the paternally derived chromosomes to give an excess of the maternal genome in the $\mathbf{M}_{2}$ generation. Unlike the radiation damage discussed above, such a mechanism would give true maternal trends regardless of the direction of the cross. It would, therefore, act in the same direction as mutational 
loss in the cross of Caligari et al., but in the opposite direction in the reciprocal cross. To estimate the contribution made by gametophytic and zygotic selection in the presence of mutational damage it is necessary to compare the $\mathrm{M}_{2}$ generations derived from reciprocal crosses. An experiment of this kind, which will also provide further information about the direct effects of radiation damage is currently in progress.

In conclusion, it is worthwhile to consider the implications of our findings for the mechanism of gene transfer which Pandey favours. Like Snape et al., we are unable to find any evidence to support Pandey's proposals. Instead we appear to be observing the more conventional effects of radiation, which are less likely to have a useful application. Only if gametophytic selection is found to be very severe is it likely that heavy irradiation of pollen could be developed as a plant breeding technique. Pandey has described his $M_{1}$ plants as being euploid, fertile, and largely maternal, in which case they are clearly different to ours. Although we have used species of the same genus as Pandey our species are self-compatible and we are therefore unable to use the mentor pollen effect used by Pandey. We have also used radiation doses considerably lower than those Pandey used, because we were unable to obtain seed from higher doses. The possibility exists that an unusual mechanism is occurring which is peculiar to the conditions of Pandey's experiments. The details and usefulness of such a mechanism are still to be determined.

Acknowledgements. We are indebted to Professor J. L. Jinks for much useful advice and critical comment. We would also like to acknowledge two SERC studentships (to C.P.W. and I.M.D.) and financial support from the ARC which enabled this work to be carried out.

\section{REFERENCES}

CALIGARI, P. D. S., INGRAM, N. R. AND JiNKS, J. L., 1981. Gene transfer in Nicotiana rustica by means of irradiated pollen. I. Unselected progenies. Heredity, 47, 17-26.

DEVREUX, M., DONINI, B. AND SCARASCIA-MUGNOZZA, G. T., 1972. Genetic effects of gametophyte irradiation in barley. II. Frequency of types of mutations induced. Radiation Botany, 12, 87-98.

DONINI, B., DEVREUX, M. AND SCARASCIA-MUGNOZZA, G. T., 1970. Genetic effects of gametophyte irradiation in barley. I. Seedling mutants. Radiation Botany, 10, 79-86.

ENGV1LD, K. C., 1980. On gene transfer from radiation inactivated pollen of Nicotiana. Incompatibility Newsletter, 13, 111-113.

GERSTEL, D. U., BURNS, J. A. AND BURK, L. G., 1978. Cytoplasmic male sterility in Nicotiana, restoration of fertility, and the nucleolus. Genetics, $89,157-169$.

GOODSPEED, T. H., 1929. Cytological and other features of variant plants produced from X-rayed sex cells of $N$. tabacum. Botanical Gazette, 87, 565-582.

IVANOV, M. A., 1938. Experimental production of haploids in N. rustica. Genetica, 20, 295-386.

PANDEY, K. K., 1975. Transformation, incompatibility and plant improvement. Incompatibility Newsletter, 6, 91-121.

PANDEY, K. K., 1978. Gametic gene transfer in Nicotiana by means of irradiated pollen. Genetica, 49, 53-69.

PANDEY, K. K., 1980. Further evidence for egg transformation in Nicotiana. Heredity, 45, 17-31.

PANDEY, K. K. AND PHUNG, M., 1982. Hertwig effect in plants: induced parthenogenesis through the use of irradiated pollen. Theoretical and Applied Genetics, 62, 295-300.

POWELl, W., CALIGARI, P. D. S. AND HAYTER, A. M., 1983. The use of pollen irradiation in barley breeding. Theoretical and Applied Genetics, 65, 73-76.

SNAPE, J. W., PARKER, B. B., SIMPSON, E., AINSWORTH, C. C., PAYNE, P. I. AND LAW, C. N., 1983. The use of irradiated pollen for differential gene transfer in wheat (Triticum aestivum). Theoretical and Applied Genetics, 65, 103-111. 